\title{
A partial classification of the blowups of the singularities in a composite membrane problem
}

\author{
Ivan Blank \\ Worcester Polytechnic Institute \\ Worcester, MA 01609 \\ E-mail: blanki@wpi.edu
}

\begin{abstract}
We give a short overview of a blowup method and describe results which are obtained when it is applied to an eigenvalue optimization problem which arises in the study of composite membranes.
\end{abstract}

We want to create a membrane with prescribed shape and mass out of given materials (with different densities) in such a way that the fundamental frequency of the resulting membrane is minimized. In [CGIKO] it is shown that the following mathematical problem is slightly more general than the physical problem just stated. We assume that we are given a domain $\Omega \subset \mathbb{R}^{2}$ which is bounded, connected, and has a Lipschitz boundary, and we are given numbers $\alpha>0$ and $A \in[0,|\Omega|]$ (where $|\Omega|$ denotes the Lebesgue 2-dimensional measure of $\Omega$ ). We let $\chi_{D}$ be the characteristic function of $D$, and $\lambda_{\Omega}(\alpha, D)$ be the minimum eigenvalue of the problem

$$
\begin{aligned}
-\Delta u+\alpha \chi_{D} u=\lambda u & \text { in } \Omega \\
u=0 & \text { on } \partial \Omega,
\end{aligned}
$$

and we define

$$
\Lambda_{\Omega}(\alpha, A):=\inf _{|D|=A, D \subset \Omega} \lambda_{\Omega}(\alpha, D) .
$$

Physically speaking, $D$ is where we use one material and $D^{c}$ is where we use the other material. The fact that we are taking a fixed area for $D$ corresponds to our assumption that the mass of the final membrane has been prescribed. Stated very simply the question becomes, "How will $D$ arrange itself to minimize the first eigenvalue?" If $D$ is a minimizer for Equation (2), and $u$ satisfies Equation (1), then $(u, D)$ is called an optimal pair (or solution). Chanillo and others (see [CGIKO] and [CGK]) have studied some of the qualitative properties of these solutions. They have shown that if we normalize $u$ by requiring $\|u\|_{L^{2}}=1$ and $u \geq 0$, then the set $D$ has the form $\{x: u(x)<t\}$ for some $t>0$. They also show that $\partial D^{c}$ is real-analytic at any point where $\nabla u \neq 0$. 
Because of the analyticity of the boundary at points where $\nabla u \neq 0$, the main obstacle to proving a Hausdorff estimate on the boundary is the lack of a classification of points where the gradient of $u$ vanishes. So we assume that we are at such a point, and to simplify notation we will assume that boundary point is the origin. By taking $w(x):=u(x)-t$, we will have a function which satisfies the structure conditions which we introduce below. (Although we have stated this problem in two dimensions, the only part of our argument which will require us to be in two dimensions will be the exact classification of the blowups. The method presented will give nontrivial blowups for all cases in all dimensions. This method is flexible enough to be applicable to other problems. See [B2].)

Remark. In a recent series of papers (see [W1] and [W2] for example), G.S. Weiss has found many applications of a monotonicity formula which he introduced in [W1], and there are some similarities between his formula and the use of the estimate (10) in this paper. In fact, it seems that the monotonicity formula of Weiss, and the technique which Spruck employed in [S] contain the same idea expressed in different variables. Within [W2] Weiss studied the problem

$$
\Delta w=f \chi_{\{w>0\}}-g \chi_{\{w<0\}}
$$

where

$$
f, g \in C^{\alpha}, \quad f \geq 0, \text { and } f+g>0 .
$$

For solutions of (3) under the assumptions (4) it turns out that there is always a quadratic bound at any point where $w$ and its gradient vanish, so there is no reason to apply the method of this paper to that situation. The solutions of our eigenvalue problem (more precisely $t-u(x)$ ) will satisfy an equation like (3), but with $f \geq 0$, and $f+g<0$.

In terms of the blowups done here, it is useful to compare our eigenvalue problem with the obstacle problem. In the obstacle problem after a change of variables, one arrives at the following setting:

$$
w \geq 0, \quad w(0)=0, \quad|\nabla w(0)|=0, \Delta w(x)=\chi_{\{w>0\}}(x) f(x) \text { in } B_{1} .
$$

In this setting, it is known (see $[\mathrm{CK}]$ ) that there is a universal quadratic bound which gives enough compactness to guarantee that the quadratic rescaling

$$
w_{\epsilon}(x):=\epsilon^{-2} w(\epsilon x)
$$

will converge to a nontrivial global solution. At that point classification of these global blowup limits can be used to study questions of local regularity of the free boundary. (See [B1] for example.)

In our setting, we have not established a quadratic bound on $w$ at zero and we cannot guarantee that the standard quadratic rescaling will converge. 
In this situation trying to get useful information from some sort of blowup becomes a much more difficult task. To give an idea of the problem, suppose that $w$ does not have a quadratic bound on any sequence of radii converging to zero, and suppose that we do a rescaling of the form

$$
w_{n}(x):=\gamma_{n} w\left(\epsilon_{n} x\right),
$$

where $\epsilon_{n} \downarrow 0$, and $\gamma_{n}$ is chosen to keep some norm of $w_{n}$ fixed. We will call a blowup of this form an inhomogeneous blowup to distinguish it from the more usual quadratic blowup where the $\gamma_{n}$ are exactly $\epsilon_{n}^{-2}$. (For now we may as well assume that we have fixed the sup norm of $w_{n}$ on the unit ball to be 1.) Because $w$ does not have a quadratic bound, the renormalization will lead to the estimate $\Delta w_{n} \rightarrow 0$ and standard regularity and compactness results allow us to conclude that a subsequence of the $w_{n}$ converges to a harmonic function $W$ on any compact subset of $B_{1}$. The problem is that the norm which we fix at 1 can "concentrate" on $\partial B_{1}$ and we can have $W \equiv 0$.

Now we describe a method which yields a successful blowup argument which leads to a classification of the possible singularities of the solution. For a function $w$ with bounded Laplacian and with $w(0)=|\nabla w(0)|=0$, we distinguish between three possibilities:

1. If there exists a constant $C$ such that $|w(x)| \leq C|x|^{2}$, then we say that $w$ has quadratic asymptotics and call this the quadratic case.

2. If $w$ does not have quadratic asymptotics, but

$$
\liminf _{r \downarrow 0} \sup _{|x|=r} \frac{|w(x)|}{r^{2}}<\infty,
$$

then we say that $w$ has mixed asymptotics and call this the mixed case.

3. If

$$
\liminf _{r \downarrow 0} \sup _{|x|=r} \frac{|w(x)|}{r^{2}}=\infty,
$$

then we say that $w$ is strictly superquadratic and call this the strictly superquadratic case.

Any function in the second or third case above will be referred to as superquadratic.

We turn to a description of the three main ingredients of our method. The first ingredient is a theorem which produces a nontrivial blow up in either superquadratic case, and is essentially found in [BS].

1 Theorem (Blank, Shahgholian). Assume that $w$ is superquadratic, that $w(0)=|\nabla w(0)|=0$, and that $|\Delta w| \leq C$ in a neighborhood of zero. Then there exists a sequence of radii $\left\{\rho_{j}\right\}$ decreasing to zero, and another sequence $\left\{\gamma_{j}\right\}$ such that the rescalings

$$
w_{j}(x):=\gamma_{j} w\left(\rho_{j} x\right)
$$

converge to a nontrivial homogeneous harmonic polynomial of degree 2 on all of $\mathbb{R}^{n}$. 
The next ingredient is a change of variables and a theorem which in the quadratic case is essentially due to Spruck. The change of variables is given by

$$
\psi(s, \theta):=\frac{w(r, \theta)}{r^{2}}, \text { with } s:=-\log r,
$$

where $(r, \theta)$ are the usual polar coordinates, and the theorem we need is the following:

2 Theorem (Spruck). Assume that $w(0)=|\nabla w(0)|=0$, that (with $\psi$ defined exactly as in Equation (9))

$$
\int_{S^{n-1}} \int_{1}^{\infty} \psi_{s}^{2} d s d \theta \leq C
$$

that $|\Delta w| \leq C$, and that $\left\{\rho_{j}\right\}$ is a sequence converging to zero such that

$$
\sup _{j} \sup _{x \in \partial B_{\rho_{j}}} \frac{|w(x)|}{|x|^{2}} \leq C \text { and } \sup _{j} \sup _{x \in \partial B_{\rho_{j}}} \frac{|\nabla w(x)|}{|x|} \leq C .
$$

Then a subsequence of the quadratic rescaling

$$
w_{j}(x):=\rho_{j}^{-2} w\left(\rho_{j} x\right)
$$

converges to a function which is homogeneous of degree two.

Remark. To motivate the change of variables and some of the corresponding structure conditions, observe that after a separation of variables in polar coordinates a Laplacian will lead to an Euler equation for the function in $r$. At that point to solve an Euler equation standard ODE textbooks will suggest the logarithmic change of variables to yield a simple constant coefficient second order ODE.

Equation (10) is the main structure condition to verify in order to apply the method here, and it holds for our problem outlined above as long as we are not in the strictly superquadratic case. (Although the $\rho_{j}$ 's given to us in a mixed case only satisfy the first part of Equation (11), we can use elliptic regularity to get both parts if we are willing to shrink the $\rho_{j}$ 's slightly.) It is also in the proof that Equation (11) will imply Equation (10) that the fact that Euler equations become constant coefficient equations under the change of variables $s:=-\log r$ comes into play very strongly. (For more details, see [B2].) After a constant coefficient equation is found for $\psi(s, \theta)$ we multiply by $\psi_{s}(s, \theta)$ and then do a computation which is reminiscent of standard energy estimates for constant coefficient second order ordinary differential equations.

At this point in the mixed case by applying Theorem (1) we have an inhomogeneous blow up to a harmonic polynomial along one sequence of radii, 
and by applying Theorem (2) along a quadratically bounded sequence, we will have a quadratic blow up to a function which is homogeneous of degree two. It turns out that, we can classify the quadratic blow up limit, and, not surprisingly, it will have different properties from the inhomogeneous blow up limit obtained along the superquadratic sequence. By looking at the equations satisfied by the blowup limits, we get the following picture in the membrane problem in two dimensions. (See [B2].)

\section{Quadratic Limit}

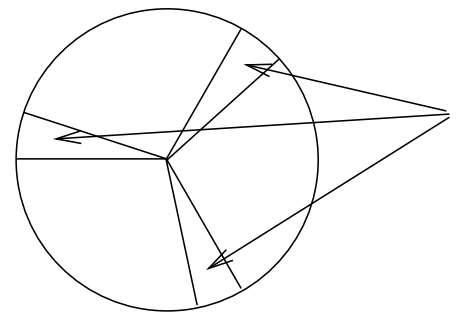

Inhomogeneous Limit

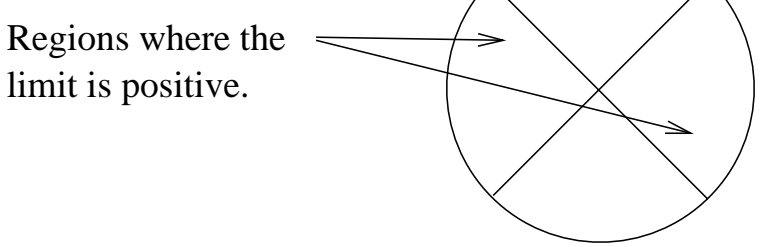

The density of the positivity set is exactly $50 \%$ in the superquadratic case, and something strictly less than $50 \%$ in the quadratic case. It is now a simple matter to produce an interlacing sequence $\left\{r_{n}\right\}$, such that the density of the positivity set along this sequence is not compatible with either blow up. Now either the function has a quadratic bound along this sequence, in which case we can use Theorem (2) to come to a contradiction, or there exists a subsequence of the $\left\{r_{n}\right\}$ (which we still call $\left\{r_{n}\right\}$ ) such that

$$
\lim _{n \rightarrow \infty} \sup _{x \in \partial B_{r_{n}}} \frac{|w(x)|}{|x|^{2}}=\infty .
$$

It is for this case where we would like to do an inhomogeneous blow up to come to a contradiction, but we cannot apply Theorem (1) because that theorem does not allow for an arbitrary choice of the radii. In other words, the $\left\{r_{n}\right\}$ of the interlacing radii are not necessarily the $\left\{\rho_{n}\right\}$ given by that theorem. The following theorem is the final ingredient of the method, and it eliminates the mixed case in our composite membrane problem. (See [B2] for the proof.)

3 Theorem (Nontrivial Inhomogeneous Blow Up). Assume that $\Delta w \in$ $L^{\infty}$, that $w(0)=|\nabla w(0)|=0$, and that with the definition of $\psi$ as above we satisfy the structure condition:

$$
\int_{S^{n-1}} \int_{0}^{\infty} \psi_{s}^{2} d s d \theta \leq C
$$

Finally, assume that a sequence $\left\{t_{j}\right\}$ is given which satisfies

$$
\lim _{j \rightarrow \infty} \int_{B_{1}}\left(\frac{w\left(t_{j} x\right)}{t_{j}^{2}}\right)^{2} d x=+\infty .
$$


Then there exists a subsequence of the $t_{j}$ (still called $t_{j}$ ), and a sequence $\ell_{j} \rightarrow$ $\infty$ such that the rescalings $w_{j}(x):=w\left(t_{j} x\right) /\left(t_{j}^{2} \ell_{j}\right)$ converge to a nontrivial harmonic polynomial of degree two.

Remark. The sudden switch from superquadratic behavior as we have defined it above to a superquadratic behavior in an $L^{2}$ norm sense is harmless in our application because of elliptic regularity theory. (If supremums become large, then $L^{2}$ norms have to become large.)

We summarize this information in the following theorem:

4 Theorem (Asymptotics and Classification). Assume $u$ is a solution of our membrane problem in dimension 2, assume $w(x)=u(x)-t$, and assume that $w(0)=|\nabla w(0)|=0$. Then either $w$ has a quadratic bound, or $w$ is strictly superquadratic. In particular, the pictures on the previous page are the only possibilities for the blowup limits. (More quantitative statements can be found in [B2].)

\section{References}

[B1] I. Blank, Sharp results for the regularity and stability of the free boundary in the obstacle problem, Indiana Univ. Math. J. 50(2001), no. 3, 10771112 .

[B2] I. Blank, Eliminating mixed asymptotics in obstacle type free boundary problems, to appear in Comm. PDE.

[BS] I. Blank and H. Shahgholian, Boundary regularity and compactness for overdetermined problems, Ann. Scuola. Norm. Sup. Pisa. Cl. Sci., 2(2003), no. 4, 787-802.

[CK] L.A. Caffarelli and D. Kinderlehrer, Potential methods in variational inequalities, J. Analyse Math., 37(1980), 285-295.

[CGIKO] S. Chanillo, D. Grieser, M. Imai, K. Kurata, and I. Ohnishi, Symmetry breaking and other phenomena in the optimization of eigenvalues for composite membranes, Comm. Math. Phys., 214(2000), no. 2, 315-337.

[CGK] S. Chanillo, D. Grieser, and K. Kurata, The free boundary problem in the optimization of composite membranes. Differential geometric methods in the control of partial differential equations, Contemp. Math., 268(2000), 61-81.

[S] J. Spruck, Uniqueness in diffusion model of population biology, Comm. P.D.E., 8(1983), 1605-1620.

[W1] G.S. Weiss, Partial regularity for weak solutions of an elliptic free boundary problem, Comm. P.D.E., 23(1998), 439-455.

[W2] G.S. Weiss, An obstacle-problem-like equation with two phases: pointwise regularity of the solution and an estimate of the Hausdorff dimension of the free boundary, Interfaces and Free Boundaries, 3(2001), 121-128. 